\title{
The 24-h clinical microbiology service is essential for patient management
}

\author{
Joseph M Blondeau*,1,2 \& Evgeny A Idelevich ${ }^{3}$ \\ ${ }^{1}$ Department of Clinical Microbiology, Royal University Hospital \& Saskatchewan Health Authority; Saskatoon, Saskatchewan, \\ Canada \\ ${ }^{2}$ Departments of Microbiology \& Immunology, Pathology \& Ophthalmology, University of Saskatchewan, Saskatoon, \\ Saskatchewan, Canada \\ ${ }^{3}$ Institute of Medical Microbiology, University Hospital Münster, Münster, Germany \\ *Author for correspondence: Tel.: +1 306655 6943; Fax: +1 306655 6947; joseph.blondeau@saskhealthauthority.ca
}

\section{“optimal patient care requires access to necessary laboratory testing including clinical microbiology. A rethinking of hours of operation is required to shorten time to accurate result reporting."}

First draft submitted: 14 August 2018; Accepted for publication: 15 October 2018; Published online: 14 November 2018

Keywords: hours of operation • medical microbiology • technology

Multidrug resistant bacteria, superbugs, outbreaks, intensive and critical care medicine, infection prevention and control, public health, emergency medicine, antimicrobial stewardship and others rely on accurate and timely testing in clinical microbiology laboratories. For many years, it was accepted that results from clinical microbiology could take days, thereby often questioning its clinical versus academic value. One can easily argue that a delayed result has a lesser impact directly on the patient than does a more timely result which could influence management decisions earlier in the disease process. For example, viral serology where acute and convalescent (2-3 weeks later) sera reveals a fourfold rise in antibody titer provided a clinical diagnosis but to a patient that was no longer ill in most cases. Advances in diagnostic technology in clinical microbiology now allow for clinically useful results more timely and in some situations, in real time.

Considerable debate is ongoing regarding the hours of operation of clinical microbiology laboratories, the additional costs of such and the clinical impact on patient care. Additionally, ancillary (and important) programs (as mentioned) such as Infection Prevention and Control and Antimicrobial Stewardship rely on accurate and rapid pathogen identification and delays in results could influence containment of bacterial spread and incorrect, poor or suboptimal empiric antibiotic therapy. The dramatic changes that have occurred in clinical microbiology laboratories over the past decade with amazing advancements in technology have changed the time-to-reporting of clinically important results to be close to real time. Maximum benefits for patient care require laboratories to be open around the clock. Dokouhaki and Blondeau previously commented on advances in diagnostic technologies and the impact on clinical practice [1].

Technological advances in clinical microbiology laboratories cannot be overstated. Continuous-monitoring blood culture instruments have shortened the time to detection of positive blood cultures when compared with earlier generation machines where trays of bottles needed to be loaded into the instrument (multiple times/day) to be read. Improvements in blood culture media $[2,3]$ to recover common and less common pathogens including fastidious and nutritionally deficit strains and Haemophilus, Aggregatibacter (previously Actinobacillus), Cardiobacterium, Eikenella, Kingella (HACEK) group organisms have allowed shorter incubation times (i.e., 5-7 days) to reporting of a negative result. Prolonged and excessive incubation times (in days/weeks) are not supported by the peerreviewed literature [4-6]. Semi-automated technology for organism identification and antimicrobial susceptibility testing (AST) (i.e., VITEK ${ }^{\circledR}$ 2, Microscan, Phoenix) impacted work flow and expanding data bases extended the genus and species of organism identified and susceptibility tested. Antigen and antibody agglutination reactions for organism speciation were quick, often inexpensive and fit well in workflow. Often these tests could be used as 'quick tests' and avoided full organism identification by other technologies that could delay time to reporting

Future Medicine 
(i.e., agglutination reactions for Streptococcus species; Staphylococcus aureus co-agglutination reactions and more). Fluorescent microscopy improved sensitivity and specificity with viral pathogens, Mycobacterium tuberculosis and Pneumocystis jiroveci. Molecular diagnostics (probes, PCR, sequencing) provided for same day (often within hours for PCR) reporting and also improved sensitivity and specificity albeit at an increased cost. Matrix-assisted laser desorption ionization - time of flight (MALDI-TOF) technology has and is redefining clinical microbiology laboratories worldwide. This technology is operationally inexpensive (expensive acquisition costs). MALDI-TOF determines the organism spectrogram, compares it to a vast data base and organism identification occurs. The process is rapid (seconds) and currently most organisms identified by MALDI-TOF are from isolated colonies growing on agar plates. Direct identification of organisms from positive blood cultures and perhaps other specimens has reduced time to result by another day [7]. There is little doubt speed and accuracy has reduced time to reporting of organism identification to minutes from hours to days. Rapid and accurate organism identification, along with up-to-date hospital antibiograms allows more confidence with empiric or organism-specific antimicrobial therapy and impacts therapeutic guideline recommendations. Furthermore, introduction of MALDI-TOF in clinical microbiology has indirectly caused rethinking in other diagnostic areas. For example, understanding that bacteria do not need to be grown overnight in order to be successfully identified [8], led to the realization that the shorter incubated cultures from solid media can simultaneously be used for inoculation of AST instruments [9]. Thus, both identification and AST results can be generated 1 day earlier without any additional cost just by the re-organization of laboratory workflow [10].

At three recent excellent ESCMID workshops on MALDI-TOF (Basel, Switzerland, March 2017), next generation sequencing (Herzliyya, Israel, November 2017) and most recently endocarditis and endovascular infection (Münster, Germany, March 2018), rapid diagnostics in clinical microbiology with advanced technology have been discussed and debated. The arguments on the benefits of advanced technology have been repeatedly made by many different speakers. Idelevich commented during his presentation on "rapid microbiological diagnosis of blood stream infections" and the concept of 'microbiologistics' impacting turnaround time. It is well known that pre-analytical factors impact time to reporting of results. Some of the pre-analytical factors include time to specimen collection from when physician or practitioner order was placed, transport to in-hospital laboratory versus transport to a referral laboratory, batch specimens transport, porter service versus pneumatic tubes and so on. In exploring delays in specimen transport, substantial time delays occur even in situations where the laboratories are not far from the clinical units. Oddly but not surprisingly, a prime reason for significant delays from specimen collection to processing in the laboratory is hours of operation of the clinical microbiology laboratory [11-13]. So, we now have this interesting dichotomy - advanced technology allowing accurate and faster results in clinical microbiology but laboratories may only be open for approximately $50 \%$ of the time and arguably sick patients may require diagnostic tests 24-h a day and certainly benefit from more timely reporting of results. Clearly, the importance of biochemistry and hematology to emergency, surgery and intensive care medicine has been recognized for decades but the same has not been true for clinical microbiology - largely due to technology limitations and delays in reporting of clinically useful management decision influencing results.

In our province (Saskatchewan) and elsewhere in Canada and North America, recent accreditation requirements were for 24-h monitoring of blood culture specimens in order to reduce delays in reporting of positive blood cultures including Gram stain results. As such, our service has medical laboratory technologists providing testing 24-h a day and a clinical/medical microbiologist is always providing 'on call' service including consultation - again $24 \mathrm{~h}$ a day, 7 days a week year round.

Our internal policy requires positive blood culture results (and other critical results, i.e., positive cerebral spinal fluid or septic joint results) be phoned to a physician responsible for the patient regardless of the time of day or night. Medical laboratory technologists in Canada are trained to perform and interpret test results based on protocols and criteria established by the clinical microbiology leadership.

Elsewhere in the world, a Gram stain on a positive blood culture, for example, requires sign off by a clinical/medical microbiologist before reporting. Such requirements would negatively impact reporting time during off hours (i.e., $7 \mathrm{pm}-7 \mathrm{am}$ ). In our institution, consultation with the clinical/medical microbiologist is only done prior to reporting of results for cases where interpretation of findings is more complicated and a more detailed conversation with the attending physician is required. As well, in our institution, the 'on call' microbiologist reviews results before reporting a sexually transmitted pathogen on a child and usually communicates directly with the attending physician and public health. 
A common comment regarding 24-h clinical microbiology laboratory service is increased costs. Costs of services can be divided between staffing (highest cost), technology and consumables. In our experience, hours of operation does not increase the number of specimens submitted but does alter specimen receiving thereby impacting workflow.

Our center in Saskatoon, SK, Canada is a university medical center providing clinical microbiology services for three acute care hospitals and community-based physicians in the areas of bacteriology, virology, mycology, parasitology, serology and molecular diagnostics. We process up to 1400 patients' specimens/day. Our 24-h service is staffed with dedicated clinical microbiology technologists. In smaller regional centers performing blood cultures, 24-h coverage for positive blood cultures is provided by technologists from chemistry or hematology that have been cross-trained to perform and interpret Gram stains and subculture to plated media. Investigations beyond this are performed by the 'day' staff. More complex patients or specimens or both are most often referred or transferred to our larger medical centers.

Riest et al. (more than 20 years ago) when comparing continuous-reading blood culture instruments with older versions commented that, "no time benefit for detection of positive blood cultures is gained with continuously measuring systems, if loading and processing of vials is organized discontinuously, as in our laboratory" [14]. In Europe, most of clinical microbiology laboratories are not staffed in the evening and night hours to insert blood culture bottles into automated incubators and perform Gram stain and subculture when blood culture signals positive. This discontinuous workflow causes time delays of up to $12 \mathrm{~h}$ prior to incubation start and another time delay of up to $12 \mathrm{~h}$ before positive blood culture can be processed. Interestingly, with such workflow when the incubation of batched blood culture bottles is started in the morning, many positive blood cultures signal in the evening and night hours, when staff is not available to process them. Indeed, a recent study found that the majority (70\%) of blood culture bottles were flagged positive by the automated system outside the laboratory's service hours [15]. Clearly, current practice does not fulfill criteria as urgent diagnostics and does not meet the needs of effective sepsis management. A 24/7 microbiological service is essential for sepsis diagnostics and joined efforts should be made by microbiologists, clinicians and managers to establish this service. Nevertheless, unfortunately it cannot be realistically expected that the current practice will promptly and broadly change all around the world. Therefore, alternative diagnostic principles can be developed to better match current practice without 24/7 staffed laboratories. Such diagnostic systems can be based on inoculation of specimens (including blood) onto solid media directly at patient's bed, followed by utilization of transportation and storage times for cultivation [16].

Where are we going in terms of further advances? At the 2018 ECCMID meeting in Madrid, Spain, rapid AST was a hot topic for discussion. Convincing literature reports that more seriously ill patients treated with the wrong antibiotic die more often than those treated with the correct agent. Some current multiplex PCR assays detect resistance markers such as methicillin-resistant $S$. aureus, carbapenemase-producing Enterobacteriaceae, producers of extended-spectrum $\beta$-lactamases, etc. and such information helps with early avoidance or switching from agents to which these organisms would be resistant. But such testing does not determine resistance caused by other mechanisms as well as susceptibility for agents that remain active against certain pathogens. Conventional susceptibility testing could take $24-48 \mathrm{~h}$ - a substantial delay - arguably during the more critical time of acute infection in a moderately to critically ill patient. A shortened turnaround time is necessary and essential.

In summary, optimal patient care requires access to necessary laboratory testing including clinical microbiology. A rethinking of hours of operation is required to shorten time to accurate result reporting.

\section{Financial \& competing interests disclosure}

EA Idelevich is a co-author of patent applications in diagnostic areas. The authors have no other relevant affiliations or financial involvement with any organization or entity with a financial interest in or financial conflict with the subject matter or materials discussed in the manuscript apart from those disclosed.

No writing assistance was utilized in the production of this manuscript.

\section{References}

1. Dokouhaki P, Blondeau JM. Advances in laboratory diagnostic technologies in clinical microbiology and what this means for clinical practice. Clin. Pract. 9(4), 347-352 (2012).

2. Zadroga R, Williams DN, Gottschall R et al. Comparison of 2 blood culture media shows significant differences in bacterial recovery for patients on antimicrobial therapy. Clin. Infect. Dis. 56(6), 790-797 (2013). 
3. Fiori B, D'inzeo T, Di Florio V et al. Performance of two resin-containing blood culture media in detection of bloodstream infections and in direct matrix-assisted laser desorption ionization-time of flight mass spectrometry (MALDI-TOF MS) broth assays for isolate identification: clinical comparison of the BacT/Alert Plus and Bactec Plus systems. J. Clin. Microbiol. 52(10), 3558-3567 (2014).

4. Forward KR. An evaluation of extended incubation time with blind subculture of blood cultures in patients with suspected endocarditis. Can. J. Infect. Dis. Med. Microbiol. 17(3), 186-188 (2006).

5. Weinstein MP. Emerging data indicating that extended incubation of blood cultures has little clinical value. Clin. Infect. Dis. 41(11), 1681-1682 (2005).

6. Petti CA, Bhally HS, Weinstein MP et al. Utility of extended blood culture incubation for isolation of Haemophilus, Actinobacillus, Cardiobacterium, Eikenella, and Kingella organisms: a retrospective multicenter evaluation. J. Clin. Microbiol. 44(1), 257-259 (2006).

7. La Scola B, Raoult D. Direct identification of bacteria in positive blood culture bottles by matrix-assisted laser desorption ionisation time-of-flight mass spectrometry. PLoS ONE 4(11), e8041 (2009).

8. Idelevich EA, Schule I, Grunastel B, Wullenweber J, Peters G, Becker K. Rapid identification of microorganisms from positive blood cultures by MALDI-TOF mass spectrometry subsequent to very short-term incubation on solid medium. Clin. Microbiol. Infect. 20(10), 1001-1006 (2014).

9. Idelevich EA, Schule I, Grunastel B, Wullenweber J, Peters G, Becker K. Acceleration of antimicrobial susceptibility testing of positive blood cultures by inoculation of Vitek 2 cards with briefly incubated solid medium cultures. J. Clin. Microbiol. 52(11), 4058-4062 (2014).

10. Idelevich EA, Becker K. Identification and susceptibility testing from shortly incubated cultures accelerate blood culture diagnostics at no cost. Clin. Infect. Dis. 62(2), 268-269 (2016).

11. Ronnberg C, Mildh M, Ullberg M, Ozenci V. Transport time for blood culture bottles: underlying factors and its consequences. Diagn. Microbiol. Infect. Dis. 76(3), 286-290 (2013).

12. Idelevich EA, Silling G, Niederbracht $Y$ et al. Impact of multiplex PCR on antimicrobial treatment in febrile neutropenia: a randomized controlled study. Med. Microbiol. Immunol. (Berl). 204(5), 585-592 (2015).

13. Kerremans JJ, Verboom P, Stijnen T et al. Rapid identification and antimicrobial susceptibility testing reduce antibiotic use and accelerate pathogen-directed antibiotic use. J. Antimicrob. Chemother. 61(2), 428-435 (2008).

14. Riest G, Linde HJ, Shah PM. Comparison of BacT/Alert and BACTEC NR 860 blood culture systems in a laboratory not continuously staffed. Clin. Microbiol. Infect. 3(3), 345-351 (1997).

15. Kock R, Wullenweber J, Horn D, Lanckohr C, Becker K, Idelevich EA. Implementation of short incubation MALDI-TOF MS identification from positive blood cultures in routine diagnostics and effects on empiric antimicrobial therapy. Antimicrob. Resist. Infect. Control 6(12), 1-7 (2017).

16. Idelevich EA, Grunastel B, Peters G, Becker K. Direct blood culturing on solid medium outperforms an automated continuously monitored broth-based blood culture system in terms of time to identification and susceptibility testing. New Microbes New Infect. 10, 19-24 (2016). 